\title{
Multistage iterations and solvability of linear Cauchy problems
}

\author{
Natalia Dilnaya and Andrei Rontó
}




\title{
MULTISTAGE ITERATIONS AND SOLVABILITY OF LINEAR CAUCHY PROBLEMS
}

\author{
NATALIA DILNAYA AND ANDREI RONTÓ
}

[Received: September 10, 2003 and, in revised form, October 6, 2003]

\begin{abstract}
New general unique solvability conditions of the Cauchy problem for many-dimensional systems of linear functional differential equations are established.

Mathematics Subject Classification: 34K06, 34K10

Keywords: Functional-differential, Cauchy problem, unique solvability, differential inequality, optimal condition
\end{abstract}

\section{INTRODUCTION}

We are interested in conditions sufficient for the unique solvability of the $n$-dimensional initial value problem of the form

$$
\begin{gathered}
u^{\prime}(t)=(\ell u)(t)+f(t), \quad t \in[a, b], \\
u(\tau)=c,
\end{gathered}
$$

where $-\infty<a<b<+\infty, u:[a, b] \rightarrow \mathbb{R}^{n}, n \in \mathbb{N}$, for arbitrary $c \in \mathbb{R}^{n}$ and $f \in L\left([a, b], \mathbb{R}^{n}\right)$. In (1.1), $\ell: C\left([a, b], \mathbb{R}^{n}\right) \rightarrow L\left([a, b], \mathbb{R}^{n}\right)$ is a linear operator which is assumed to be $(\vec{\sigma}, \tau)$-positive in the following sense.

Definition 1.1. [1,3] An operator $\ell: C\left([a, b], \mathbb{R}^{n}\right) \rightarrow L\left([a, b], \mathbb{R}^{n}\right)$ is said to be $(\vec{\sigma}, \tau)$-positive with some $\vec{\sigma} \in\{-1,1\}^{n}$ and $\tau \in[a, b]$ if the relation

$$
u(t) \geq_{\vec{\sigma}} 0 \text { for all } t \in[a, b],
$$

implies that

$$
(\ell u)(t) \operatorname{sign}(t-\tau) \geq_{\vec{\sigma}} 0 \quad \text { for a. e. } t \in[a, b] .
$$

Here, the symbol $\geq_{\vec{\sigma}}$ stands for the partial ordering of the space $\mathbb{R}^{n}$ generated by the cone $\sigma_{1} \mathbb{R}_{+} \times \sigma_{2} \mathbb{R}_{+} \times \cdots \times \sigma_{n} \mathbb{R}_{+}$, i. e.,

$$
\mathbb{R}^{n} \ni u=\left(u_{k}\right)_{k=1}^{n} \geq_{\vec{\sigma}} 0 \text { if, and only if } \sigma_{k} u_{k} \geq 0 \text { for all } k=1,2, \ldots, n .
$$

The work of the second author was supported in part by NATO Science Fellowships Programme for the Czech Republic, Grant 7/2003. The author is also grateful to the staff of the Mathematical Institute of the Czech Academy of Sciences for their hospitality and kind attention. 
In a similar way, we define the corresponding strict inequality:

$$
\mathbb{R}^{n} \ni u=\left(u_{k}\right)_{k=1}^{n}>_{\vec{\sigma}} 0 \text { if, and only if } \sigma_{k} u_{k}>0 \text { for all } k=1,2, \ldots, n \text {. }
$$

By a solution of problem (1.1), (1.2), as usual, an absolutely continuous function $u:[a, b] \rightarrow \mathbb{R}^{n}$ is meant which possesses property (1.2) at the point $\tau$ and satisfies equation (1.1) almost everywhere on $[a, b]$.

Remark 1.2. As noted in [3], a $(\vec{\sigma}, \tau)$-positive linear operator from $C\left([a, b], \mathbb{R}^{n}\right)$ to $L\left([a, b], \mathbb{R}^{n}\right)$ is always bounded.

For the Cauchy problem (1.1), (1.2), we obtain new versions of the general unique solvability conditions established in $[1,3]$. The main idea here, as the title of the paper suggests, is to use certain "multistage iterations," in terms of which the main assumptions of the theorems are formulated.

\section{UNIQUE SOLVABILITY OF PROBLEM (1.1), (1.2)}

To formulate the theorems on the Cauchy problem (1.1), (1.2), we need to introduce some auxiliary functions related to the homogeneous problem

$$
\begin{gathered}
u^{\prime}(t)=(\ell u)(t), \quad t \in[a, b], \\
u(\tau)=0 .
\end{gathered}
$$

2.1. Multistage iterations for the homogeneous problem. With the homogeneous problem (2.1), (2.2), which corresponds, in a natural way, to the original Cauchy problem (1.1), (1.2), we associate the sequence of functions $\left\{y_{k} \mid k=0,1,2, \ldots\right\} \subset$ $C\left([a, b], \mathbb{R}^{n}\right)$ determined by the recurrence relation

$$
y_{k}(t):=\sum_{i=1}^{r} \alpha_{i} \int_{\tau}^{t}\left(\ell y_{k-i}\right)(s) d s, \quad t \in[a, b], \quad k \geq r,
$$

where $\alpha_{1}, \alpha_{2}, \ldots, \alpha_{r}$ are certain fixed non-negative constants.

Here, $r \in \mathbb{N}$ is fixed, and $y_{0}, y_{1}, \ldots, y_{r-1}$ are arbitrary absolutely continuous functions from $[a, b]$ to $\mathbb{R}^{n}$ chosen so that

$$
y_{k}(t) \geq_{\vec{\sigma}} 0, \quad t \in[a, b], k=0,1, \ldots, r-1,
$$

and

$$
y_{k}(\tau)=0, \quad k=0,1, \ldots, r-1 .
$$

Remark 2.1. In the case where $r=1$ and $\alpha_{1}=1$, equality (2.3) takes the form

$$
y_{k}(t)=\int_{\tau}^{t}\left(\ell y_{k-1}\right)(s) d s, \quad t \in[a, b], \quad k \in \mathbb{N},
$$


and thus coincides with the sequence used, e. g., in [1-3]. Formula (2.6) defines the standard iteration sequence used in studies of the uniqueness of the trivial solution of the integral functional equation

$$
y(t)=\int_{\tau}^{t}(\ell y)(s) d s, \quad t \in[a, b],
$$

which, obviously, is equivalent to the homogeneous problem (2.1), (2.2).

2.2. General theorems. The following general theorem on the solvability of problem (1.1), (1.2) is true.

Theorem 2.2. Let the operator $\ell$ in equation (1.1) be $(\vec{\sigma}, \tau)$-positive for some $\vec{\sigma} \in$ $\{-1,1\}^{n}$. Assume also that one can specify some integers $r$ and $m, m \geq r \geq 1$, a real number $\varrho \in(1,+\infty)$, some constants $\left\{\beta_{k}\right\}_{k=0}^{m} \subset[0,+\infty)$ and $\left\{\alpha_{i}\right\}_{i=1}^{r} \subset[0,+\infty)$, and certain absolutely continuous vector-functions $y_{0}, y_{1}, \ldots, y_{r-1}$ satisfying conditions (2.4), (2.5), and the relation

$$
\sum_{k=0}^{r-1} \beta_{k} y_{k}(t)>_{\vec{\sigma}} 0 \quad \text { for all } t \in[a, b] \backslash\{\tau\}
$$

such that the differential functional inequality

$$
\begin{aligned}
{\left[\sum_{k=0}^{r-1} \beta_{k} y_{k}^{\prime}(t)+\sum_{k=0}^{m-1}\left(\sum_{v \in T_{r, m}(k)} \beta_{v+k} \alpha_{v}-\varrho \beta_{k}\right)\left(\ell y_{k}\right)(t)\right.} & \\
& \left.-\varrho \beta_{m}\left(\ell y_{m}\right)(t)\right] \operatorname{sign}(t-\tau) \geq_{\vec{\sigma}} 0
\end{aligned}
$$

is satisfied for almost every t from $[a, b]$, where, by definition,

$$
T_{r, m}(k):=\{v \in \mathbb{N} \mid v \leq r \leq v+k \leq m\}
$$

for $r \in \mathbb{N}, m \geq r$, and $k=0,1, \ldots, m-1$.

Then the Cauchy problem (1.1), (1.2) has a unique solution $u(\cdot)$ for arbitrary $f \in L\left([a, b], \mathbb{R}^{n}\right)$ and $c \in \mathbb{R}^{n}$, and this solution is representable as the uniformly convergent functional series

$$
u(t)=\tilde{f}(t)+\int_{\tau}^{t}(\ell \tilde{f})(s) d s+\int_{\tau}^{t} \ell\left(\int_{\tau}^{\cdot}(\ell \tilde{f})(\xi) d \xi\right)(s) d s+\ldots, \quad t \in[a, b],
$$

where, by definition,

$$
\tilde{f}(t):=c+\int_{\tau}^{t} f(s) d s, \quad t \in[a, b] .
$$

Moreover, if the function $f$ and vector $c$ in (1.1), (1.2) satisfy the additional condition

$$
\int_{\tau}^{t} f(s) d s \geq_{\vec{\sigma}}-c \quad \text { for all } t \in[a, b],
$$


then the unique solution $u(\cdot)$ of this problem satisfies relation (1.3).

Remark 2.3. It is not difficult to show that the set $T_{r, m}(k)$, where $k=0,1, \ldots, m-$ 1 , consists of those integers $v$ which satisfy the inclusion $v \in\{r, r+1, \ldots, m\} \cap$ $\{k+1, k+2, \ldots, k+r\}$, or, in other words,

$$
T_{r, m}(k)=\{v \in \mathbb{N} \mid \max \{r, k+1\} \leq v \leq \min \{m, k+r\}\}
$$

for $k=0,1, \ldots, m-1$. Therefore, the differential inequality (2.8) can be rewritten in the form

$$
\begin{aligned}
{\left[\sum_{k=0}^{r-1} \beta_{k} y_{k}^{\prime}(t)+\sum_{k=0}^{m-1}\left(\sum_{v=\max \{r, k+1\}}^{\min \{m, k+r\}} \beta_{v+k} \alpha_{v}-\varrho \beta_{k}\right)\left(\ell y_{k}\right)(t)\right.} & \\
& \left.-\varrho \beta_{m}\left(\ell y_{m}\right)(t)\right] \operatorname{sign}(t-\tau) \geq_{\vec{\sigma}} 0 .
\end{aligned}
$$

It seems, however, that form (2.8) is more convenient for applications.

Note that, due to the inequality $m \geq r$, for $k=0,1,2, \ldots, m-1$, we have

$$
\max \{r, k+1\} \leq m, \quad \max \{r, k+1\} \leq k+r
$$

and, hence, by (2.13), the set $T_{r, m}(k)$ is non-empty for any $k=0,1, \ldots, m-1$.

Remark 2.4. Condition (2.7) implies, in particular, that

$$
\sum_{k=0}^{r-1} \beta_{k}>0,
$$

and, therefore, we can always take 1 for one of the non-negative coefficients $\beta_{0}, \beta_{1}$, $\ldots, \beta_{r-1}$ in Theorem 2.2. It is handier, however, to keep all these coefficients unfixed.

Remark 2.5. Theorem 2.2 implies, in particular, Theorem 1 from [1]. Both these statements, as we shall see in Section 3, are implied by Theorem 1 from [3]. This means that the theorems mentioned are, in fact, equivalent, although their corresponding assumptions are rather different from one another.

Remark 2.6. Similarly to [3], one can show that condition (2.8) is optimal in the sense that the assertion of Theorem 2.2, generally speaking, is not true if (2.8) is assumed with $\varrho=1$.

The assumptions of the following version of Theorem 2.2 are somewhat simpler.

Theorem 2.7. Let the operator $\ell$ in equation (1.1) be $(\vec{\sigma}, \tau)$-positive for some $\vec{\sigma} \in$ $\{-1,1\}^{n}$. Assume also the existence of a natural number $r$, real number $\varrho \in(1,+\infty)$, some non-negative constants $\left\{\alpha_{i}\right\}_{i=1}^{r}$ and $\left\{\beta_{k}\right\}_{k=0}^{r}$, and certain absolutely continuous 
vector-functions $y_{0}, y_{1}, \ldots, y_{r-1}$ satisfying conditions (2.4), (2.5), and (2.7) such that the differential functional inequality

$$
\left[\sum_{k=0}^{r-1} \beta_{k} y_{k}^{\prime}(t)+\sum_{k=0}^{r-1}\left(\beta_{r} \alpha_{r-k}-\varrho \beta_{k}\right)\left(\ell y_{k}\right)(t)-\varrho \beta_{r}\left(\ell y_{r}\right)(t)\right] \operatorname{sign}(t-\tau) \geq_{\vec{\sigma}} 0
$$

is satisfied for almost every $t$ from $[a, b]$.

Then the Cauchy problem (1.1), (1.2) has a unique solution $u(\cdot)$ for arbitrary $f \in L\left([a, b], \mathbb{R}^{n}\right)$ and $c \in \mathbb{R}^{n}$, and this solution is representable as the uniformly convergent functional series (2.10), where $\tilde{f}$ is the function defined by equality (2.11).

Moreover, if the function $f$ and vector $c$ in (1.1), (1.2) satisfy the additional condition (2.12), then the unique solution $u(\cdot)$ of this problem satisfies relation (1.3).

Note that the functions $y_{0}, y_{1}, \ldots, y_{r-1}$ in Theorem 2.7 are given a priori, whereas the function $y_{r}$ is constructed according to formula (2.3):

$$
y_{r}(t)=\sum_{i=1}^{r} \alpha_{i} \int_{\tau}^{t}\left(\ell y_{r-i}\right)(s) d s, \quad t \in[a, b]
$$

2.3. Corollaries. Here, we present several corollaries derived from Theorems 2.2 and 2.7 .

Corollary 2.8. Let the operator $\ell$ in equation (1.1) be $(\vec{\sigma}, \tau)$-positive for some $\vec{\sigma} \in$ $\{-1,1\}^{n}$. Assume also the existence of an $r \in \mathbb{N}$, a real number $\gamma \in(1,+\infty)$, some non-negative constants $\left\{\beta_{k}\right\}_{k=0}^{r-1}$, and of certain absolutely continuous vector-functions $y_{0}, y_{1}, \ldots, y_{r-1}$ satisfying conditions (2.4), (2.5), and (2.7) such that the differential functional inequality

$$
\left[\sum_{k=0}^{r-1} \beta_{k} y_{k}^{\prime}(t)-\gamma \sum_{i=1}^{r} \beta_{r-i}\left(\ell\left(\int_{\tau}\left(l y_{r-i}\right)(s) d s\right)\right)(t)\right] \operatorname{sign}(t-\tau) \geq_{\vec{\sigma}} 0
$$

holds for the functions $y_{0}, y_{1}, \ldots, y_{r-1}$ at almost every point $t$ from $[a, b]$.

Then the conclusion of Theorem 2.7 is true for problem (1.1), (1.2).

The conditions involving iteration sequences of type (2.3) admit different variations. For example, the following statement is true.

Corollary 2.9. Let the operator $\ell$ in equation $(1.1)$ be $(\vec{\sigma}, \tau)$-positive for some $\vec{\sigma} \in$ $\{-1,1\}^{n}$. Assume that there exist certain absolutely continuous functions $y_{0}, y_{1}$ : $[a, b] \rightarrow \mathbb{R}^{n}$ satisfying the conditions

$$
y_{k}(t)>_{\vec{\sigma}} 0 \text { and } y_{k}(\tau)=0 \text { for all } t \in[a, b] \backslash\{\tau\}, k=0,1,
$$

and some constants $\varrho \in(1,+\infty), m \in \mathbb{N}(m \geq 2),\left\{\alpha_{1}, \alpha_{2}\right\} \subset[0,+\infty), \alpha_{1} \alpha_{2} \neq 0$, and $\left\{\beta_{0}, \beta_{1}, \beta_{2}, \ldots, \beta_{m}\right\} \subset(0,+\infty)$ such that

$$
\alpha_{1} \beta_{k+1}+\alpha_{2} \beta_{k+2}=\varrho \beta_{k}, \quad k=1,2, \ldots, m-2,
$$


for which the relation

$$
\begin{aligned}
& {\left[\beta_{0} y_{0}^{\prime}(t)+\beta_{1} y_{1}^{\prime}(t)+\left(\alpha_{2} \beta_{2}-\varrho \beta_{0}\right)\left(\ell y_{0}\right)(t)\right.} \\
& \left.\quad+\left(\alpha_{1} \beta_{m}-\varrho \beta_{m-1}\right)\left(\ell y_{m-1}\right)(t)-\varrho \beta_{m}\left(\ell y_{m}\right)(t)\right] \operatorname{sign}(t-\tau) \geq_{\vec{\sigma}} 0
\end{aligned}
$$

holds for almost all $t \in[a, b]$, where the functions $y_{m-1}$ and $y_{m}$ are defined by the recurrence relation

$$
y_{k}(t):=\alpha_{1} \int_{\tau}^{t}\left(\ell y_{k-1}\right)(s) d s+\alpha_{2} \int_{\tau}^{t}\left(\ell y_{k-2}\right)(s) d s, \quad t \in[a, b],
$$

for $k=2,3, \ldots, m$.

Then the conclusion of Theorem 2.7 is true for problem (1.1), (1.2).

Remark 2.10. As will be seen from the proof of Corollary 2.9, instead of (2.17), the absolutely continuous functions $y_{0}$ and $y_{1}$ can be assumed to be just non-negative in the sense of relation $\geq_{\vec{\sigma}}$ (see formula (1.4)) and such that

$$
y_{0}(\tau)=y_{1}(\tau)=0 ; \quad y_{0}(t)+y_{1}(t)>_{\vec{\sigma}} 0 \quad \text { for } \quad t \in[a, b] \backslash\{\tau\},
$$

i. e., one of these functions may vanish at points different from $\tau$ whenever the other one remains non-zero.

Corollary 2.11. If $\ell$ in equation (1.1) is $(\vec{\sigma}, \tau)$-positive for some $\vec{\sigma} \in\{-1,1\}^{n}$ and, furthermore, one can specify some $\varrho \in(1,+\infty), \alpha \in(0, \varrho)$, and certain absolutely continuous vector-functions $z_{0}, z_{1}:[a, b] \rightarrow \mathbb{R}^{n}$ satisfying the conditions

$$
z_{k}(t)>_{\vec{\sigma}} 0 \text { and } z_{k}(\tau)=0 \text { for all } t \in[a, b] \backslash\{\tau\}, k=0,1,
$$

and, for almost every $t$ from $[a, b]$, the inequality

$$
\left[z_{0}^{\prime}(t)+z_{1}^{\prime}(t)-(\varrho-\alpha)\left(\ell z_{0}\right)(t)-\alpha\left(\ell z_{m-1}\right)(t)-\varrho\left(\ell z_{m}\right)(t)\right] \operatorname{sign}(t-\tau) \geq_{\vec{\sigma}} 0,
$$

where the $z_{m-1}$ and $z_{m}$ are the functions defined recursively by the relation

$$
z_{k}(t):=(\varrho-\alpha) \int_{\tau}^{t}\left(\ell z_{k-1}\right)(s) d s+\alpha \int_{\tau}^{t}\left(\ell z_{k-2}\right)(s) d s, \quad t \in[a, b],
$$

for $k=2,3, \ldots, m$, then the assertion of Theorem 2.7 is true for problem (1.1), (1.2).

The following version of Corollary 2.11 is more similar to Theorem 3.1.

Corollary 2.12. If $\ell$ in equation (1.1) is $(\vec{\sigma}, \tau)$-positive for some $\vec{\sigma} \in\{-1,1\}^{n}$ and, furthermore, there exist some $\varrho \in(1,+\infty), \alpha \in(0, \varrho)$, and certain absolutely continuous vector-functions $z_{0}, z_{1}:[a, b] \rightarrow \mathbb{R}^{n}$ satisfying conditions (2.20) and, for almost every $t$ from $[a, b]$, the inequality

$$
\left[z_{0}^{\prime}(t)+z_{1}^{\prime}(t)-(\varrho-\alpha)\left(\ell z_{0}\right)(t)-\alpha\left(\ell z_{1}\right)(t)-\varrho\left(\ell z_{2}\right)(t)\right] \operatorname{sign}(t-\tau) \geq_{\vec{\sigma}} 0,
$$


where

$$
z_{2}(t):=(\varrho-\alpha) \int_{\tau}^{t}\left(\ell z_{1}\right)(s) d s+\alpha \int_{\tau}^{t}\left(\ell z_{0}\right)(s) d s, \quad t \in[a, b],
$$

then the assertion of Theorem 2.7 is true for problem (1.1), (1.2).

For the equation with deviating argument

$$
u^{\prime}(t)=\sum_{j=1}^{N} P_{j}(t) u\left(\omega_{j}(t)\right)+f(t), \quad t \in[a, b],
$$

where $f \in L\left([a, b], \mathbb{R}^{n}\right), N \in \mathbb{N},\left\{P_{j} \mid j=1,2, \ldots, N\right\} \subset L\left([a, b], G L_{n}(\mathbb{R})\right)$, and $\omega_{j}:[a, b] \rightarrow[a, b], j=1,2, \ldots, N$, are measurable functions, one can establish, e. g., the following statement.

Corollary 2.13. Assume that, for a certain $\vec{\sigma} \in\{-1,1\}^{n}$, the functions $P_{j}:[a, b] \rightarrow$ $G L_{n}(\mathbb{R})$ and $\omega_{j}:[a, b] \rightarrow[a, b], j=1,2, \ldots, N$, in equation (2.23) satisfy the inequalities

$$
\sum_{v=1}^{N} P_{v}(t) \vec{\sigma} \operatorname{sign}(t-\tau) \geq_{\vec{\sigma}} 0 \quad \text { for a. e. } t \in[a, b]
$$

and, furthermore, the condition

$$
\begin{aligned}
& \sum_{i=1}^{r} \beta_{r-i} \sum_{j=1}^{N} P_{j}(t) \int_{\tau}^{\omega_{j}(t)} \sum_{v=1}^{N} P_{v}(s) g\left|\omega_{v}(s)-\tau\right|^{q^{r-i}} d s \cdot \operatorname{sign}(t-\tau) \\
& \quad \leq_{\vec{\sigma}} \alpha \sum_{k=0}^{r-1} \beta_{k} q_{k}|t-\tau|^{q_{k}-1} g \quad \text { for } a . \text { e. } t \in[a, b]
\end{aligned}
$$

holds with some $\left\{\beta_{k} \mid k=0,1, \ldots, r-1\right\} \subset[0,+\infty),\left\{q_{k} \mid k=0,1, \ldots, r-1\right\} \subset[1,+\infty)$, $\alpha \in(0,1)$, and $g \in \mathbb{R}^{n}, g>_{\vec{\sigma}} 0$.

Then, for arbitrary $f$ from $L\left([a, b], \mathbb{R}^{n}\right)$ and $c \in \mathbb{R}^{n}$, the initial value problem (2.23), (1.2) has a unique solution $u(\cdot)$, and this solution is representable as the uniformly convergent series

$$
\begin{aligned}
u(t)= & \tilde{f}(t)+\sum_{k_{1}=1}^{N} \int_{\tau}^{t} P_{k_{1}} u\left(\xi_{1}\right) \tilde{f}\left(\omega_{k_{1}}\left(\xi_{1}\right)\right) d \xi_{1} \\
& +\sum_{k_{1}=1}^{N} \int_{\tau}^{t} P_{k_{1}}\left(\xi_{1}\right) \int_{\tau}^{\omega_{k_{1}}\left(\xi_{1}\right)} \sum_{k_{2}=1}^{N} P_{k_{2}}\left(\xi_{2}\right) \tilde{f}\left(\omega_{k_{2}}\left(\xi_{2}\right)\right) d \xi_{2} d \xi_{1}+\ldots, \quad t \in[a, b],
\end{aligned}
$$

with the function $\tilde{f}:[a, b] \rightarrow \mathbb{R}^{n}$ given by formula (2.11). Moreover, under the additional condition (2.12), the solution of problem (2.23), (1.2) satisfies inequality (1.3). 


\section{Proofs}

We need the following theorem from [3].

Theorem 3.1. [3] Let us suppose that the linear operator $\ell$ in equation $(1.1)$ is $(\vec{\sigma}, \tau)$ positive for some $\vec{\sigma} \in\{-1,1\}^{n}$. Assume also that there exist a number $\varrho \in(1,+\infty)$ and an absolutely continuous function $y:[a, b] \rightarrow \mathbb{R}^{n}$ such that

$$
\begin{gathered}
y(\tau)=0, \\
y(t)>_{\vec{\sigma}} 0 \quad \text { for all } t \in[a, b] \backslash\{\tau\},
\end{gathered}
$$

and, furthermore, the functional differential inequality

$$
\left[y^{\prime}(t)-\varrho(\ell y)(t)\right] \operatorname{sign}(t-\tau) \geq_{\vec{\sigma}} 0
$$

is satisfied for almost every $t$ from $[a, b]$.

Then the Cauchy problem (1.1), (1.2) is uniquely solvable for arbitrary $c \in \mathbb{R}^{n}$ and $f \in L\left([a, b], \mathbb{R}^{n}\right)$. The unique solution $u(\cdot)$ of problem (1.1), (1.2) is, moreover, representable as the uniformly convergent functional series (2.10).

If, in addition, $c$ and $f$ satisfy condition (2.12), then the solution $u(\cdot)$ mentioned possesses property (1.3).

The idea of proof of the results stated in Sections 2.2 and 2.3 is to construct a suitable solution of the differential inequality (3.3) by using some or other properties of the "multistage" iteration sequence (2.3).

3.1. Proof of Theorem 2.2. Let us fix certain absolutely continuous vector-functions $\left\{y_{k}\right\}_{k=0}^{r-1}:[a, b] \rightarrow \mathbb{R}^{n}$ and construct the corresponding functions $\left\{y_{k}\right\}_{k=r}^{m}:[a, b] \rightarrow \mathbb{R}^{n}$ according to formula (2.3). We consider the case where

$$
m \geq r .
$$

Introduce the function

$$
y(t):=\sum_{k=0}^{m} \beta_{k} y_{k}(t), \quad t \in[a, b],
$$

with the coefficients $\left\{\beta_{k}\right\}_{k=0}^{m} \subset[0,+\infty)$ determined by the assumptions of the theorem. Note that, in view of (2.4), assumption (2.7) implies that (2.14) holds.

Let us show that, under our assumptions, function (3.5) satisfies inequality (2.8). Indeed, by virtue of (3.5), the corresponding function

$$
w_{\varrho, y}:=y^{\prime}-\varrho \ell y
$$

has the form

$$
w_{\varrho, y}=\sum_{k=0}^{m} \beta_{k}\left[y_{k}^{\prime}-\varrho \ell y_{k}\right],
$$


whence, by (3.4),

$$
\begin{aligned}
w_{\varrho, y} & =\sum_{k=0}^{r-1} \beta_{k}\left[y_{k}^{\prime}-\varrho \ell y_{k}\right]+\sum_{k=r}^{m} \beta_{k}\left[y_{k}^{\prime}-\varrho \ell y_{k}\right] \\
& =\sum_{k=0}^{r-1} \beta_{k} y_{k}^{\prime}+\sum_{k=r}^{m} \beta_{k} y_{k}^{\prime}-\varrho \sum_{k=0}^{m} \beta_{k} \ell y_{k} .
\end{aligned}
$$

Note that the first sum in (3.7) contains the given functions $y_{0}, y_{1}, \ldots, y_{r-1}$ only.

In view of formula (2.3) for the functions $y_{r}, y_{r+1}, \ldots$, we have

$$
y_{k}^{\prime}(t):=\sum_{i=1}^{r} \alpha_{i}\left(\ell y_{k-i}\right)(t), \quad t \in[a, b], \quad k \geq r,
$$

and, therefore, equality (3.7) can be brought to the form

$$
w_{\varrho, y}=\sum_{k=0}^{r-1} \beta_{k} y_{k}^{\prime}+\sum_{k=r}^{m} \beta_{k} \sum_{i=1}^{r} \alpha_{i} \ell y_{k-i}-\varrho \sum_{k=0}^{m} \beta_{k} \ell y_{k} .
$$

Now we need the following technical lemma.

Lemma 3.2. For arbitrary vectors $x_{0}, x_{1}, \ldots, x_{m-1}$ from $\mathbb{R}^{n}$, the equality

$$
\sum_{k=r}^{m} \beta_{k} \sum_{i=1}^{r} \alpha_{i} x_{k-i}=\sum_{j=0}^{m-1} \gamma_{j} x_{j}
$$

holds, where the coefficients $\gamma_{0}, \gamma_{1}, \ldots, \gamma_{m-1}$ are given by the formulae

$$
\gamma_{k}:=\sum_{v \in T_{r, m}(k)} \beta_{v+k} \alpha_{v}, \quad k=0,1, \ldots, m-1,
$$

and the sets $T_{r, m}(k), k=0,1, \ldots, m-1$, are defined by equality (2.9).

Proof of Lemma 3.2. It is clear from the inequalities $1 \leq i \leq r \leq k \leq m$ that the expression

$$
\sum_{k=r}^{m} \beta_{k} \sum_{i=1}^{r} \alpha_{i} x_{k-i}
$$

is, in fact, a linear combination of the values $x_{0}, x_{1}, \ldots, x_{m-1}$. It therefore remains to determine the corresponding coefficients.

Since equality (3.9) should be satisfied for arbitrary values of $x_{0}, x_{1}, \ldots, x_{m-1}$, we can put, in particular,

$$
x_{j}:=\delta_{j, s}, \quad j=0,1, \ldots, m-1,
$$

where $s$ is an arbitrary integer between 0 and $m-1$, and $\delta_{j, s}$ is the Kronecker symbol,

$$
\delta_{j, s}= \begin{cases}1 & \text { for } j=s \\ 0 & \text { otherwise. }\end{cases}
$$


In this case, (3.9) has the form

$$
\sum_{k=r}^{m} \beta_{k} \sum_{i=1}^{r} \alpha_{i} \delta_{k-i, s}=\gamma_{s}
$$

It is easy to see that

$$
\sum_{i=1}^{r} \alpha_{i} \delta_{k-i, s}= \begin{cases}\alpha_{k-s} & \text { if } k-s \in\{1,2, \ldots, r\} \\ 0 & \text { if } k-s \notin\{1,2, \ldots, r\}\end{cases}
$$

and, hence,

$$
\sum_{k=r}^{m} \beta_{k} \sum_{i=1}^{r} \alpha_{i} \delta_{k-i, s}=\sum_{k: r \leq k \leq m \text { and }} \beta_{1 \leq k-s \leq r} \alpha_{k-s} .
$$

In view of (3.11), the change of variable $v=k-s$ brings (3.12) to the form

$$
\sum_{v: 1 \leq v \leq r \leq v+s \leq m} \beta_{v+s} \alpha_{v}=\gamma_{s}
$$

The last relation, according to the definition of the sets $T_{r, m}(s), s=0,1, \ldots, m-1$, can be rewritten as $\sum_{v \in T_{r, m}(s)} \beta_{v+s} \alpha_{v}=\gamma_{s}$, which is nothing but (3.10). This leads us immediately to the required equality (3.9) for arbitrary $x_{0}, x_{1}, \ldots, x_{m-1}$.

Returning now to expression (3.6), we conclude that, in view of Lemma 3.2, formula (3.8) can be rewritten as

$$
\begin{aligned}
w_{\varrho, y} & =\sum_{k=0}^{r-1} \beta_{k} y_{k}^{\prime}+\sum_{k=0}^{m-1} \gamma_{k} \ell y_{k}-\varrho \sum_{k=0}^{m} \beta_{k} \ell y_{k} \\
& =\sum_{k=0}^{r-1} \beta_{k} y_{k}^{\prime}+\sum_{k=0}^{m-1}\left(\gamma_{k}-\varrho \beta_{k}\right) \ell y_{k}-\varrho \beta_{m} \ell y_{m},
\end{aligned}
$$

where $\gamma_{0}, \gamma_{1}, \ldots, \gamma_{m-1}$ are given by relation (3.10). In view of (3.10), equality (3.13) is equivalent to the relation

$$
w_{\varrho, y}=\sum_{k=0}^{r-1} \beta_{k} y_{k}^{\prime}+\sum_{k=0}^{m-1}\left(\sum_{v \in T_{r, m}(k)} \beta_{v+k} \alpha_{v}-\varrho \beta_{k}\right) \ell y_{k}-\varrho \beta_{m} \ell y_{m} .
$$

Therefore, assumption (2.8) guarantees that function (3.6) satisfies the condition

$$
w_{\varrho, y}(t) \operatorname{sign}(t-\tau) \geq_{\vec{\sigma}} 0 \quad \text { for a. e. } t \in[a, b],
$$

i. e., the functional differential inequality (3.3) holds for the function $y$ given by (3.5).

We have thus constructed a solution, $y$, of the differential inequality (3.3). It will be possible to apply Theorem 3.1 if we show that, under our assumptions, the solution mentioned possesses properties (3.1) and (3.2). We prove this by using the following lemma. 
Lemma 3.3. Assume that the operator $\ell: C\left([a, b], \mathbb{R}^{n}\right) \rightarrow L\left([a, b], \mathbb{R}^{n}\right)$ is $(\vec{\sigma}, \tau)$ positive with respect to certain $\vec{\sigma} \in\{-1,1\}^{n}$ and $\tau \in[a, b]$. Then, for arbitrary continuous functions $\left\{y_{k}\right\}_{k=0}^{r-1}:[a, b] \rightarrow \mathbb{R}^{n}$ satisfying conditions (2.4) and (2.5), the corresponding functions $y_{r}, y_{r+1}, \ldots$ defined by formulae (2.3) also satisfy condition (2.4):

$$
y_{k}(t) \geq_{\vec{\sigma}} 0, \quad t \in[a, b], \quad k \geq r .
$$

Moreover, each of these functions vanishes at the point $\tau$.

Proof of Lemma 3.3. The first assertion is derived from the definition of functions (2.3) similarly to the proof of Lemma 1 from [1]. The important difference is that, in our case, we need to make use of the non-negativeness of the coefficients $\alpha_{1}, \alpha_{2}, \ldots, \alpha_{r}$ in formula (2.3) for the functions $y_{r}, y_{r+1}, \ldots$. We omit the details here.

The property

$$
y_{k}(\tau)=0 \quad \text { for all } k=0,1,2, \ldots, m
$$

is obvious from condition (2.5) and formula (2.3).

Application of Lemma 3.3 allows us to claim that, in view of the $(\vec{\sigma}, \tau)$-positiveness of the operator $\ell$, the inequality

$$
\beta_{k} y_{k}(t) \geq_{\vec{\sigma}} 0, \quad t \in[a, b], \quad k=0,1, \ldots, m,
$$

is satisfied because all $\beta_{k}, k=0,1, \ldots, m$, are non-negative. It follows from (3.14) that

and, hence,

$$
\sum_{k=0}^{m} \beta_{k} y_{k}(t)-\sum_{k=0}^{r-1} \beta_{k} y_{k}(t)=\sum_{k=r}^{m} \beta_{k} y_{k}(t) \geq_{\vec{\sigma}} 0
$$

for every $t$ from $[a, b]$.

$$
\sum_{k=0}^{m} \beta_{k} y_{k}(t) \geq_{\vec{\sigma}} \sum_{k=0}^{r-1} \beta_{k} y_{k}(t)
$$

Inequality (3.15) yields

$$
y(t)=\sum_{k=0}^{m} \beta_{k} y_{k}(t) \geq_{\vec{\sigma}} \sum_{k=0}^{r-1} \beta_{k} y_{k}(t), \quad t \in[a, b],
$$

whence, by virtue of assumption (2.7), we obtain

$$
y(t) \geq_{\vec{\sigma}} \sum_{k=0}^{r-1} \beta_{k} y_{k}(t)>_{\vec{\sigma}} 0, \quad t \in[a, b] \backslash\{\tau\},
$$

i. e., $y$ satisfies condition (3.2).

Thus, we have shown that function (3.5) satisfies the differential functional inequality (3.3) and possesses properties (3.1) and (3.2), i. e., the assumptions of Theorem 3.1 are satisfied. Application of Theorem 3.1 leads us to the assertion required. 
3.2. Proof of Theorem 2.7. To obtain the assertion of Theorem 2.7, it suffices to apply Theorem 2.2 with $m=r$ and take into account the equalities

$$
T_{r, r}(k)=\{r-k\}, \quad k=0,1, \ldots, r-1 .
$$

3.3. Proof of Corollary 2.8. We shall apply Theorem 2.7. For this purpose, let us put

$$
\varrho=\sqrt{\gamma}, \quad \beta_{r}=1,
$$

and

$$
y_{r}(t):=\sqrt{\gamma} \sum_{i=1}^{r} \beta_{r-i} \int_{\tau}^{t}\left(l_{r-i}\right)(s) d s, \quad t \in[a, b] .
$$

This function, as is easy to see, coincides with the function $y_{r}$ defined by formula (2.3) where

$$
\alpha_{i}=\beta_{r-i} \sqrt{\gamma}, \quad i=1,2, \ldots, r .
$$

It remains to notice that, in view of (3.17) and (3.18), the relations

$$
\beta_{r} \alpha_{r-k}-\varrho \beta_{k}=0, \quad k=0,1, \ldots, r-1,
$$

are true and, therefore, the corresponding differential inequality (2.15) takes the form

$$
\left[\sum_{k=0}^{r-1} \beta_{k} y_{k}^{\prime}(t)-\varrho \beta_{r}\left(l y_{r}\right)(t)\right] \operatorname{sign}(t-\tau) \geq_{\vec{\sigma}} 0 .
$$

Application of Theorem 2.7 completes the proof.

3.4. Proof of Corollary 2.9. We shall show that, under the conditions assumed, the differential inequality (2.8) is true and Theorem 2.2 is applicable.

For an arbitrary integer $k$ such that $0 \leq k \leq m-1$, a number $v$ belongs to the set $T_{2, m}(k)$ if, and only if either $v=1$ or $v=2$, i. e.,

$$
\bigcup_{k=0}^{m-1} T_{2, m}(k) \subset\{1,2\} .
$$

Recall that, by assumption, $m \geq 2$.

More precisely, $1 \in T_{2, m}(k) \Leftrightarrow 1 \leq k \leq m-1$ and $2 \in T_{2, m}(k) \Leftrightarrow 0 \leq k \leq m-2$. Thus, the sets $T_{2, m}(k), k=0,1, \ldots, m-1$, have the following structure:

$$
T_{2, m}(k)= \begin{cases}\{2\} & \text { for } k=0, \\ \{1,2\} & \text { for } k=1,2, \ldots, m-2, \\ \{1\} & \text { for } k=m-1 .\end{cases}
$$


Therefore, with these values of the parameters, the differential inequality (2.8) of Theorem 2.2 has the form

$$
\begin{aligned}
{\left[\beta_{0} y_{0}^{\prime}(t)+\beta_{1} y_{1}^{\prime}(t)+\right.} & \left(\alpha_{2} \beta_{2}-\varrho \beta_{0}\right)\left(\ell y_{0}\right)(t) \\
& +\sum_{k=1}^{m-2}\left(\alpha_{1} \beta_{k+1}+\alpha_{2} \beta_{k+2}-\varrho \beta_{k}\right)\left(\ell y_{k}\right)(t) \\
& \left.+\left(\alpha_{1} \beta_{m}-\varrho \beta_{m-1}\right)\left(\ell y_{m-1}\right)(t)-\varrho \beta_{m}\left(\ell y_{m}\right)(t)\right] \operatorname{sign}(t-\tau) \geq_{\vec{\sigma}} 0 .
\end{aligned}
$$

Recall that the numbers $\beta_{1}, \beta_{2}, \ldots, \beta_{m}$, and $\alpha_{1}, \alpha_{2}$, by assumption, satisfy relations (2.18).

It follows from (2.18) that the inner sum in (3.19) is equal to zero, i. e., in this case, (2.8) coincides with (2.19). It now remains to apply Theorem 2.2, which leads us immediately to the assertion desired.

3.5. Proof of Corollary 2.11. First of all note that sequence (2.22) is a particular case of (2.3) with a suitable choice of coefficients. More precisely,

$$
z_{k}=y_{k}, \quad k=2,3, \ldots
$$

with $y_{0}=z_{0}, y_{1}=z_{1}, r=2$, and

$$
\alpha_{2}=\alpha, \quad \alpha_{1}=\alpha .
$$

We can apply Corollary 2.9. Indeed, let us set

$$
\beta_{k}=\beta, \quad k=0,1, \ldots, m,
$$

where $\beta$ is a certain positive constant. Since, by (3.20), $\alpha_{1}+\alpha_{2}=\varrho$, it is obvious that relations (2.18) are satisfied. Inequality (2.19) in our case has form (2.21).

3.6. Proof of Corollary 2.12. It suffices to put $m=2$ in Corollary 2.11.

3.7. Proof of Corollary 2.13. Let us put $\gamma=\frac{1}{\alpha}$ and, for $k=0,1, \ldots, r-1$,

$$
y_{k}(t)=|t-\tau|^{q_{k}}, \quad t \in[a, b] .
$$

Functions (3.21), obviously, satisfy condition (2.7) with arbitrary non-negative constants $\beta_{0}, \beta_{1}, \ldots, \beta_{r-1}$ such that $\sum_{k=0}^{r-1} \beta_{k}>0$.

By using the formulae

$$
y_{k}^{\prime}(t)=q_{k}|t-\tau|^{q_{k}-1} \operatorname{sign}(t-\tau), \quad t \in[a, b], k=0,1, \ldots, r-1,
$$

it is easy to verify that, in this case, (2.25) coincides with inequality (2.16). The $(\vec{\sigma}, \tau)$-positiveness of the linear operator

$$
C\left([a, b], \mathbb{R}^{n}\right) \ni u \longmapsto \ell u:=\sum_{v=1}^{N} P_{v}(\cdot) u\left(\omega_{v}(\cdot)\right), \quad t \in[a, b],
$$


under condition (2.24), is a consequence of Lemma 2 from [1]. Therefore, the required assertion follows from Corollary 2.8.

\section{REFERENCES}

[1] Dilnaya, N. Z. and Ronto, A. N.: Some new solvability conditions of the Cauchy problem for systems of linear functional differential equations. Ukrain. Math. J., 56 (2004). (to appear).

[2] НАKL, R., Lomtatidze, A., AND PŮžA, B.: On nonnegative solutions of first order scalar functional differential equations. Mem. Differential Equations Math. Phys., 23 (2001), 51-84.

[3] Ronto, A. N.: Exact solvability conditions of the Cauchy problem for systems of linear firstorder functional differential equations determined by $\left(\sigma_{1}, \sigma_{2}, . . \sigma_{n} ; \tau\right)$-positive operators. Ukrain. Math. J., 55 (2003), No. 11, 1561-1588.

\section{Authors' Addresses}

\section{Natalia Dilnaya:}

Institute of Mathematics, National Academy of Sciences of Ukraine, 3 Tereschenkovskaya, 01601 KIEv, UKraine

E-mail address: dilna@imath.kiev.ua

\section{Andrei Rontó:}

Institute of Mathematics, National Academy of Sciences of Ukraine, 3 Tereschenkovskaya, 01601 KIEv, UKRAINE

Current address: Mathematical Institute, Czech Academy of Sciences, Žižkova 22, CZ-61662 Brno, Czech Republic

E-mail address: ronto@ipm.cz 